\title{
The role of medical data in efficient patient care delivery: a review
}

This article was published in the following Dove Medical Press journal: Risk Management and Healthcare Policy

\section{Kasaw Adane Mucheye Gizachew ${ }^{2}$ Semalegne Kendie ${ }^{3}$ \\ 'Unit of Quality Assurance and Laboratory Management, School of Biomedical and Laboratory Sciences, University of Gondar, Ethiopia;

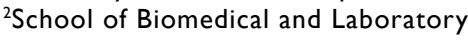 Sciences, Department of Medical Microbiology, University of Gondar, Gondar, Ethiopia; ${ }^{3}$ School of Sociology and Social Work, Department of Social Work, University of Gondar, Gondar, Ethiopia}

Background: Implementing accurate data management systems ensure safe and efficient transfer of confidential health care data. However, health care professionals overlooked their important tasks of medical data processing. Hence, using high-quality electronic health record (EHR) applications in health care is important to minimize medical errors. Therefore, this review tries to indicate the roles of EHR in advancing quality health care service provisions.

Methods: The keywords identified were EHR, EMR, medical data processing, medical data retention, medical data destruction, health care, and patient care, and a few related terms with different combinations. PubMed (National Library of Medicine), Google Scholar, and Google search engine were used to search for articles from those databases. Searching was done using boolean words "AND", "OR", and "NOT" using all [All fields] and [MeSH Terms] searching strategies.

Results: Articles were screened using the title, checked by their abstract, and the remaining related full-text materials were included or excluded by two individuals deciding its eligibility. Finally, 73 materials issued from 2013-2018 were used for qualitatively synthesizing and reconciling the idea to produce this review article.

Conclusion: Poor medical data processing systems are the key reasons for medical errors. Employing standardized data management systems reduce errors and associated sufferings. Therefore, using electronic tools in the health care institution ensures safe and efficient data management. Therefore, it is important to establish appropriate medical data management systems for efficient health care delivery.

Keywords: electronic medical data, health care data, medical data processing

\section{Background}

The mission of health care institutions - restoring patient's health - demands effective and efficient medical data for evidence-based intervention. ${ }^{1}$ Installing an appropriate health care data management system with valid case definition enables efficient data extraction, ${ }^{2}$ improves communication for clinical decision making in medical practice, ${ }^{2-8}$ and clinical research, ${ }^{9,10}$ and upgrades the quality of health care services. ${ }^{11}$ Healthcare professionals are responsive to improve recording, distributing, monitoring, and implementing preventive measures to decrease morbidity. ${ }^{12}$ This requires consistent, complete, comprehensive, and accurate information which attracts more attention in the health care industry. ${ }^{3}$

The health care industry uses a paper-based record (PBR) and/or electronic health record (EHR) system to manage patient's data. The EHR has become an integral part of medical care, ${ }^{13}$ which transforms health care service quality ${ }^{14,15}$ and improves
Correspondence: Kasaw Adane

Unit of Quality Assurance and Laboratory Management, School of Biomedical and Laboratory Sciences, University of Gondar, PO Box 196, Gondar, Ethiopia Email kswadane@gmail.com 
clinicians' satisfaction and facilitates patients' decision. ${ }^{8,16}$ Accurate information from EHR enables physicians' order entry and measures clinical validity, which in turn upgrades the quality of patient care. ${ }^{17}$ This functionality is crucial during diagnosis and therapy, ${ }^{15}$ which benefits medical and legal practices too. ${ }^{18}$

Decision-support embedded features - standardized checklists, alert signals, predictive tools, and guidelines ${ }^{1}$ - motivate and encourage health care organization leaders and persuade physicians to better utilize best practice alerts (BPAs) in a more effective and efficient way. ${ }^{19}$ In line with this, research reports backed up a position that health care practices are being transformed from PBR to EHR systems, ${ }^{17}$ although a report revealed that, in the eye care practice, EHR is less versatile for recording. ${ }^{20}$

Patient data were readily accessible and transferable ${ }^{21}$ from the EHR system. This helps to make an accurate diagnosis and decision making ${ }^{22}$ by reducing the access time and use. ${ }^{1,2}$ Notification signal flags or BPAs prompt about "what content" and "with whom" to share ${ }^{23-25}$ that trigger potential adverse events (AEs) using easily identifiable displays that alert patient records reviewers. ${ }^{26}$ This enhances patient's engagement in health care service provision ${ }^{27}$ and decisionmaking processes, ${ }^{28}$ as it builds trust ${ }^{29}$ and confidence ${ }^{30}$ that helps to identify specific and actionable adherence barriers. ${ }^{31}$ In addition, automatic email text and telephone reminders can be sent to patients in order to motivate and maximize patients compliance. ${ }^{32}$

Poor data management practices are the reasons for recurrent errors and associated injuries or death, ${ }^{33}$ which is mostly happening due to illegible $\mathrm{PBR}^{34}$ (mistakes in recording or transcribing). ${ }^{35}$

The EHR application improves the process, ${ }^{36}$ trustworthiness, safety, and efficiency of patient care delivery. ${ }^{29}$ Hence, implementing standardized policies, processes, and procedures for an appropriate health care data management system that advances the quality of health services and efficiency, ${ }^{34,37}$ avoids non-value adding activities, ${ }^{34}$ and ensures major quality and safety improvement. ${ }^{16,17,23,34}$ Therefore, this paper intends to indicate the roles of EHR in improving the quality of health care service provisions.

\section{Methods}

The keywords identified were EHR, EMR, electronic health record, electronic medical record, medical data recording, medical data processing, medical data retention, medical data destruction, health care, patient care, animal data, and plant data with different combinations. Searching was done using boolean words "AND", "OR", and "NOT".
We used [((EHR OR EHR[MeSH terms]) OR EMR) OR (EMR[MeSH terms]) OR (electronic health record) OR (electronic health record[MeSH terms]) OR (electronic medical record) OR (medical data recording[MeSH terms]) OR (medical data processing) OR (medical data processing[MeSH terms]) OR (medical data retention) OR (medical data retention[MeSH terms]) OR (medical data destruction) OR (medical data destruction[MeSH terms])] AND [((health care) OR (health care)[MeSH terms]) OR (patient care) OR (patient care)[MeSH Terms])] to search articles from PubMed and Google Scholar databases and Google search engine. Information was extracted from downloaded materials and used for qualitative synthesis.

\section{Result}

PubMed (National Library of Medicine [NLM]) databases and Google Scholar databases, as well as the Google search engine, were used for downloading published materials using EndNote $^{\circledR}$ Version $\mathrm{X}^{5}$ for Window's application. Published materials which were searched using the EndNote application were subsequently screened and checked for relevance using titles, abstracts, and full-text articles, which was done by two individuals, independently inspecting for its eligibility. From a total of 4,606 searched published materials, 73 full-text materials issued from 2013-2018 were used for the development of this review after passing the subsequent screening, selections, and checking processes. Information generated from referenced materials was qualitatively synthesized and the idea was reconciled to produce this review article. The overall study selection process is depicted in Figure 1.

\section{Discussion \\ Patient health care data management processes}

Although the health care industry is an information enterprise, its data recording practices and its data protection laws vary considerably among hospitals and countries..$^{38,39}$ The overall health care data management policies must define confidentiality and prevent reconstruction after destruction controlled by security personnel.

The document destruction policy must define the medical data retention policy and its codes of practice that must file the advantages and disadvantages of destroying or maintaining medical data. ${ }^{40}$

\section{The benefits of EHR implementation}

Implementing EHR increases the quality of services and ensures the safety of patients upon using decision-support tools result in error reduced services that increase clinicians 


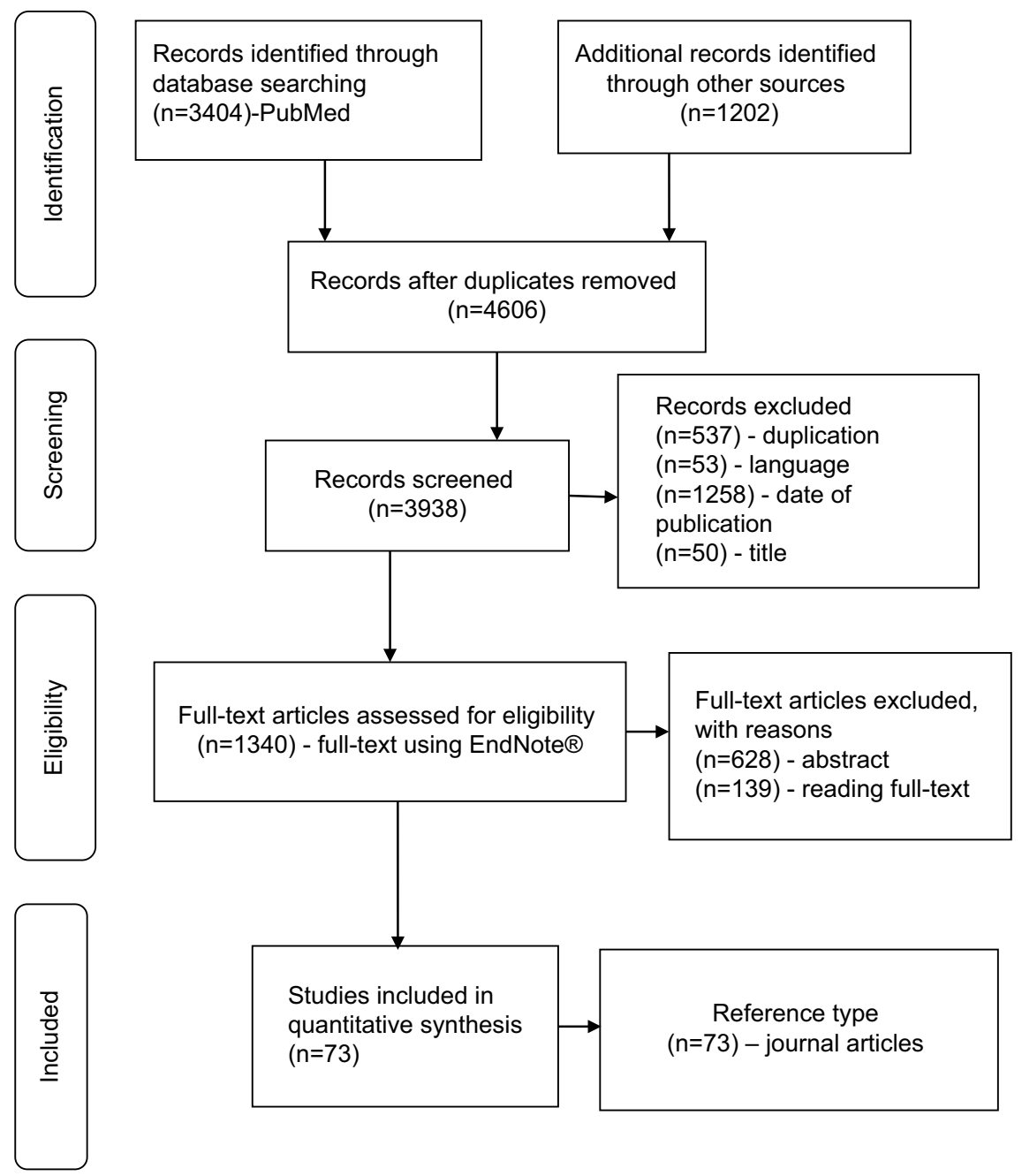

Figure I PRISMA flow diagram of article selection process.

and patient's satisfaction, which in turn increases the health care seeking-behavior of clients.

Currently, about 1,000 EHR applications are published every month ${ }^{42}$ for the purpose of increasing performance, ${ }^{41,42}$ reducing fatigue, improving accessibility, ensuring compliance, fidelity, and satisfaction, ${ }^{41,43}$ with acceptable safety gains. ${ }^{44}$

The EHR tool was implemented in the United States and the United Kingdom, which own the largest private and public health care systems in the world, respectively, and succeeded in providing quality patient care. ${ }^{45}$ It is an essential tool for the application of modern information technology that improves the quality of health care services ${ }^{46}$ consistent with medico-legal considerations. ${ }^{18}$

Accessing the EHR tool facilitates the health care delivery, ${ }^{19,24}$ made more accurate decisions, ${ }^{22}$ and contributes to the health care quality improvement and research output $t^{47,48}$ at reduced cost. ${ }^{49,50}$ The tool also ensures the safe transfer of health care data that meets the patient's expectation, ${ }^{51}$ supports the continuity of patient care ${ }^{11}$ and maintains the compliance with medication adherence. ${ }^{52,53}$ Moreover, the tool helps diabetes goal achievement, while the service delivery process is assisted from non-physician workers. ${ }^{54}$

The data generated from the EHR measure prevention, process, and outcome metric. ${ }^{55}$ Implementing high-quality EHR improves epidemic surveillance,${ }^{56}$ decreases the length of patient stay, ${ }^{40}$ achieves work efficiency ${ }^{33,40}$ by reducing non-value adding activities, ${ }^{34}$ achieves goals, ${ }^{3}$ and helps to make for timely decisions at reduced cost. ${ }^{49,57}$ The system reduces the nurses and the clerk's time spent to access data to make timely interventions. ${ }^{1}$ In its effectiveness, it ensures the quality of services at a reduced cost. ${ }^{58}$ The potential benefits of EHR are improving quality, ensuring continuity of patient care, efficiency, and positive financial return on investment. ${ }^{50}$

The effective use of EHR improves the patient's safety, ${ }^{48}$ trust, and their satisfaction on the health care system appeared orienting patients towards a health related information sources. ${ }^{59}$ Patients usually want to control how and what details to be notified when their data are accessed. ${ }^{23}$ 
The tools could be customized to notify and ensure the safe transfer of patient private confidential data, ${ }^{33}$ and they need to get protected. ${ }^{60}$

The interoperability of medical information among health care institutions increases the medical staff's understanding of the disease, diagnosis, and decision-making processes. ${ }^{61}$ The EHR allows automated disease surveillance, and helps in participation and promotion of safe and effective health care practices. ${ }^{56}$

\section{The challenges of implementing EHR}

The EHR is perceived as a "double-edged sword" as it improves quality on the one hand and increases privacy and safety risks on the other hand. ${ }^{34}$ These are important concerns of patients' for transferring their health care data..$^{23,33}$

Although its adoption rate is currently rising, EHR is found at a low rate, particularly in developing countries. ${ }^{46,62,63}$ Some of the factors for this low adoption rate include behavioral factors (lack of perceived benefits, ${ }^{28}$ poor confidence, ${ }^{64}$ dissatisfaction, ${ }^{59}$ physicians' resistance, ${ }^{65}$ lack of stakeholders interest, ${ }^{49}$ and ignorance on more advanced systems), ${ }^{60,66}$ technical factors (interoperability, ${ }^{64}$ lack of financial support or specific financial incentives, ${ }^{49}$ and lack of technology infrastructure), ${ }^{47}$ legal factors (lack of legal framework ${ }^{64}$ and lack of comprehensive EHR national policy and strategy), ${ }^{47}$ sociodemographic factors (age and education level of physicians), ${ }^{59}$ practice related factors (high skill demand ${ }^{28}$ and lack of training), ${ }^{67}$ and knowledge related factors (poor awareness).$^{64}$

The Delphi study disclosed the barriers of medical practices to implement EHR, as hindered by a myriad of intrinsic (behavioral and cognitive) and extrinsic (economic and technological) barriers when faced with the initial decision to invest in an EMR system. ${ }^{50}$

\section{Healthcare service at a distance}

Traditional telephone services were the milestones of modern telemedicine. Implementing electronic communication applications with high computational power enables the control of operations at a distance possible. Although reducing medical errors is an international agenda, physicians still commit different types of errors during manual medical data processing incurred during recording and/or fail to timely record health care data. ${ }^{35}$ Errors associated with medical data are common and costly. However, the social, spiritual, psychological, and ethical scopes of the technology, as well as the technical feasibilities of the technology, must be considered, and all stakeholders must contribute while planning and implementing new health care technologies. The PBR systems are practically more error-prone, however, the mere replacement of the system with EHR could not ensure accuracy. ${ }^{34}$ Hence, efficient processing, usage, and storage of medical data are important for both clinical and public health decisions.

\section{The future perspectives}

The promising EHR implementation systems, people, process, and product factors play an integral role in the fate of its implementation. ${ }^{11}$ The stakeholder's benefit from the systems which protect the patient's need and ensure their privacy. ${ }^{24}$ The access to accurate and complete clinical information is the main component of effective decision making. ${ }^{69}$ This is facilitated by decision-support EHR tools - BPAs - and designed for behavioral health integration with the needs of health care institutions and the benefits of improving the patient experiences, ${ }^{36}$ for instance, alcohol use. ${ }^{30}$ The system can be used to update the current condition of a patient as input to obtain a corresponding recommendation for medical tests, possible diseases, and treatment plans. ${ }^{69}$ Research indicated that the EHR "active choice" significantly increased influenza vaccination rates and ordering of colonoscopy and mammography screening services. ${ }^{8}$

The successes or challenges of voice input application can be used to transcribe doctor's dictation and facilitate the collection, indexation, storage, and retrieval processes of medical information. ${ }^{17}$ According to a study, EHR promotes services but could not favor collaborative team's culture and professionals. ${ }^{70}$

The shift in the use of EHR by the health builds trusts and presents an opportunity to monitor admission, diagnosis, and outcome to inform public health policy and service provision. ${ }^{58}$ The EHR vendors should be encouraged to incorporate social knowledge networking features into the systems. ${ }^{71}$

Authors have also identified two issues demanding the researcher's attention for more elaborated reasons for uncertainties. First, one national level research reported the adoption level of EHR as it was higher in rural practices than urban counterparts, reversing the earlier trends. ${ }^{72}$ Another similar research also reported the necessity of considering the patient's behavioral aspects while using the tool during patient rendering procedures so as to increase patient's engagement level. ${ }^{73}$ These issues may call for behavioral scientists to address this particular patient concern.

\section{Conclusion}

Medical data processing is one of the most basic tasks of the health care professionals. Computerized physician order entry applications having decision-support fields reduce 
avoidable medical errors using inbuilt memory aid. These automatic notification alert signals enable appropriate and timely intervention that ensures safer and efficient health care. The design policies of electronic technology must meet pre-stated standards and guidelines to ensure confidentiality. User-friendly technologies ensure the efficient and timely transfer of health care data for quality patient care meeting the needs of the patients and the organization.

\section{Abbreviations}

AEs, adverse events; BPAs, best practice alerts; EHR, electronic health record; EMR, electronic medical record; NLM, National Library of Medicine; PBR, paper-based record.

\section{Author contributions}

All authors contributed towards data analysis, drafting and critically revising the paper, gave final approval of the version to be published and agree to be accountable for all aspects of the work.

\section{Disclosure}

The authors report no conflicts of interest in this work.

\section{References}

1. Tierney WM, Sidle JE, Diero LO, et al. Assessing the impact of a primary care electronic medical record system in three Kenyan rural health centers. J Am Med Inform Assoc. 2016;23(3):544-552.

2. Xu Y, Li N, Lu M, et al. Development and validation of method for defining conditions using Chinese electronic medical record. $B M C M e d$ Inform Decis Mak. 2016;16(1):110.

3. Weir CR, Staggers N, Gibson B, et al. A qualitative evaluation of the crucial attributes of contextual information necessary in EHR design to support patient-centered medical home care. BMC Med Inform Decis Mak. 2015;15(1):30.

4. Weber GM, Kohane IS. Extracting Physician Group intelligence from electronic health records to support evidence based medicine. PLoS One. 2013;8(5):e64933.

5. Halpern Y, Horng S, Choi Y, Sontag D. Electronic medical record phenotyping using the anchor and learn framework. J Am Med Inform Assoc. 2016;23(4):731-740.

6. Lakin JR, Isaacs E, Sullivan E, et al. Emergency physicians' experience with advance care planning documentation in the electronic medical record: useful, needed, and elusive. J Palliat Med. 2016;19(6):632-638.

7. Bookman K, West D, Ginde A, et al. Embedded clinical decision support in electronic health record decreases use of high-cost imaging in the emergency department: embed study. Acad Emerg Med. 2017;24(7):839-845.

8. Patel MS, Volpp KG, Small DS, et al. Using active choice within the electronic health record to increase influenza vaccination rates. $J$ Gen Intern Med. 2017;32(7):790-795.

9. Aref-Eshghi E, Oake J, Godwin M, et al. Identification of dyslipidemic patients attending primary care clinics using electronic medical record (EMR) data from the Canadian primary care sentinel surveillance Network (CPCSSN) database. J Med Syst. 2017;41(3):45.

10. Deshazo JP, Hoffman MA. A comparison of a multistate inpatient EHR database to the HCUP nationwide inpatient sample. BMC Health Serv Res. 2015;15(1):384

11. Jawhari B, Keenan L, Zakus D, et al. Barriers and facilitators to electronic medical record (EMR) use in an urban slum. Int J Med Inform. 2016;94:246-254.
12. Vieira DS, Santos NC, Costa DK, et al. Recording actions to prevent child morbidity in children's health cards. Cien Saude Colet. 2016;21(7):2305-2313.

13. Vaughn VM, Linder JA. Thoughtless design of the electronic health record drives overuse, but purposeful design can nudge improved patient care. BMJ Qual Saf. 2018;27(8):583-586.

14. Tweya H, Feldacker C, Gadabu OJ, et al. Developing a point-of-care electronic medical record system for TB/HIV co-infected patients: experiences from Lighthouse trust, Lilongwe, Malawi. BMC Res Notes. 2016;9(1):146.

15. Zhang XY, Zhang P. Recent perspectives of electronic medical record systems. Exp Ther Med. 2016;11(6):2083-2085.

16. Walker E, Mcmahan R, Barnes D, Katen M, Lamas D, Sudore R. Advance care planning documentation practices and accessibility in the electronic health record: implications for patient safety. J Pain Symptom Manage. 2018;55(2):256-264.

17. Zeng X. The impacts of electronic health record implementation on the health care workforce. $N C$ Med $J$. 2016;77(2):112-114.

18. Siegel DM, Kinscherff R. Recording routine forensic mental health evaluations should be a standard of practice in the 21 st century. Behav Sci Law. 2018;36(3):373-389.

19. Chen H, Butler E, Guo Y, et al. Facilitation or hindrance: physicians' perception on Best Practice Alerts (BPA) Usage in an Electronic Health Record System. Health Commun. 2018;2:1-7.

20. Wolffsohn JS, Naroo SA, Christie C, et al. Anterior eye health recording. Cont Lens Anterior Eye. 2015;38(4):266-271.

21. Sung SF, Chen K, Wu DP, et al. Applying natural language processing techniques to develop a task-specific EMR interface for timely stroke thrombolysis: a feasibility study. Int J Med Inform. 2018;112:149-157.

22. Ben-Assuli O, Sagi D, Leshno M, Ironi A, Ziv A. Improving diagnostic accuracy using EHR in emergency departments: a simulation-based study. J Biomed Inform. 2015;55:31-40.

23. Caine K, Kohn S, Lawrence C, et al. Designing a patient-centered user interface for access decisions about EHR data: implications from patient interviews. J Gen Intern Med. 2015;30(S1):7-16.

24. Blumenthal D, Squires D. Giving patients control of their EHR data. $J$ Gen Intern Med. 2015;30(S1):42-43.

25. Milne H, Huby G, Buckingham S, et al. Does sharing the electronic health record in the consultation enhance patient involvement? A mixed-methods study using multichannel video recording and in-depth interviews in primary care. Health Expect. 2016;19(3):602-616.

26. Margham T, Symes N, Hull SA. Using the electronic health record to build a culture of practice safety: evaluating the implementation of trigger tools in one general practice. Br J Gen Pract. 2018;68(669): e279-e285.

27. El Miedany Y, El Gaafary M, El Aroussy N, et al. Toward electronic health recording: evaluation of electronic patient reported outcome Measures (e-PROMs) system for remote monitoring of early systemic lupus patients. Clin Rheumatol. 2017;36(11):2461-2469.

28. Hunter EG. Capsule commentary on Lee et al., patient perceptions of electronic medical record use by faculty and resident physicians: a mixed methods study. J Gen Intern Med. 2016;31(11):1355.

29. Ozair FF, Jamshed N, Sharma A, Aggarwal P. Ethical issues in electronic health records: a general overview. Perspect Clin Res. 2015;6(2):73-76.

30. Haroon S, Wooldridge D, Hoogewerf J, et al. Information standards for recording alcohol use in electronic health records: findings from a national consultation. BMC Med Inform Decis Mak. 2018;18(1):36.

31. Zullig LL, Curtis LH. A population health perspective on a claims and electronic health record-based tool to screen for suboptimal medication adherence. Am Heart J. 2018;197:150-152.

32. Joseph CL, Ownby DR, Zoratti E, et al. Recruitment experience for a pragmatic randomized controlled trial: using EMR initiatives and minimizing research infrastructure. Clin Res Regul Aff. 2016;33(2-4):25-32.

33. Embi PJ, Weir C, Efthimiadis EN, et al. Computerized provider documentation: findings and implications of a multisite study of clinicians and administrators. J Am Med Inform Assoc. 2013;20(4):718-726.

34. Ajami S, Bagheri-Tadi T. Barriers for adopting electronic health records (EHRs) by physicians. Acta Inform Med. 2013;21(2):129-134. 
35. Hripcsak G, Albers DJ. Next-generation phenotyping of electronic health records. JAm Med Inform Assoc. 2013;20(1):117-121.

36. Jetelina KK, Woodson TT, Gunn R, et al. Evaluation of an electronic health record (EHR) tool for integrated behavioral health in primary care. J Am Board Fam Med. 2018;31(5):712-723.

37. Top M, Yilmaz A, Karabulut E, et al. Validation of a nurses' views on electronic medical record systems (EMR) questionnaire in Turkish health system. J Med Syst. 2015;39(6):67.

38. Lujic S, Watson DE, Randall DA, Simpson JM, Jorm LR. Variation in the recording of common health conditions in routine hospital data: study using linked survey and administrative data in New South Wales, Australia. BMJ Open. 2014;4(9):e005768.

39. Vivanti A, Daly A. Important privacy considerations with electronic health record documentation. Nutr Diet. 2018;75(3):337-338.

40. Yang P, Cao Y, Liu D, Bai Y, Pan F, Xu Y. The effect of electronic medical record application on the length of stay in a Chinese General Hospital: a department- and disease-focused interrupted time-series study. J Med Syst. 2014;38(5):53.

41. Woods SS, Evans NC, Frisbee KL. Integrating patient voices into health information for self-care and patient-clinician partnerships: veterans affairs design recommendations for patient-generated data applications. J Am Med Inform Assoc. 2016;23(3):491-495.

42. Kim S, Lee K-H, Hwang H, et al. Analysis of the factors influencing healthcare professionals' adoption of mobile electronic medical record (EMR) using the unified theory of acceptance and use of technology (UTAUT) in a tertiary hospital. BMC Med Informat Decis Making. 2015;16(1):12.

43. van Drongelen A, Boot CR, Hlobil H, Smid T, van der Beek AJ. Process evaluation of a tailored mobile health intervention aiming to reduce fatigue in airline pilots. BMC Public Health. 2016;16(1):894.

44. Fernando B, Morrison Z, Kalra D, Cresswell K, Sheikh A. Approaches to recording drug allergies in electronic health records: qualitative study. PLoS One. 2014;9(4):e93047.

45. Wilson K, Khansa L. Migrating to electronic health record systems: a comparative study between the United States and the United Kingdom. Health Policy. 2018;122(11):1232-1239.

46. Zayyad MA, Toycan M. Factors affecting sustainable adoption of e-health technology in developing countries: an exploratory survey of Nigerian hospitals from the perspective of healthcare professionals. PeerJ. 2018;6(6):e4436.

47. Cowie MR, Blomster JI, Curtis LH, et al. Electronic health records to facilitate clinical research. Clin Res Cardiol. 2017;106(1):1-9.

48. Sayyah Gilani M, Iranmanesh M, Nikbin D, Zailani S. EMR continuance usage intention of healthcare professionals. Inform Health Soc Care. 2017;42(2):153-165.

49. Yoshida Y, Imai T, Ohe K. The trends in EMR and CPOE adoption in Japan under the National strategy. Int J Med Inform. 2013;82(10):1004-1011.

50. Paré G, Raymond L, de Guinea AO, et al. Barriers to organizational adoption of EMR systems in family physician practices: a mixedmethods study in Canada. Int J Med Inform. 2014;83(8):548-558.

51. Burney A, Abbas Z, Mahmood N, Arifeen Q-Ul. Prospects for mobile health in Pakistan and other developing countries. AIT. 2013;03(02):27-32.

52. Bacon TS, Fan KC, Desai MA. Electronic medical record and glaucoma medications: connecting the medication reconciliation with adherence. Clin Ophthalmol. 2016;10:221-225.

53. Yeboah-Korang A, Beig MI, Khan MQ, et al. Hepatitis C screening in commercially insured U.S. birth-cohort patients: factors associated with testing and effect of an EMR-based screening alert. J Transl Int Med. 2018;6(2):82-89.
54. Singh K, Johnson L, Devarajan R, et al. Acceptability of a decisionsupport electronic health record system and its impact on diabetes care goals in South Asia: a mixed-methods evaluation of the CARRS trial. Diabetic Med. 2018;35(12):1644-1654.

55. Cho I, Boo EH, Lee SY, Dykes PC. Automatic population of eMeasurements from EHR systems for inpatient falls. J Am Med Inform Assoc. 2018;25(6):730-738.

56. Zheng H, Gaff H, Smith G, Delisle S. Epidemic surveillance using an electronic medical record: an empiric approach to performance improvement. PLoS One. 2014;9(7):e100845.

57. Leightley D, Chui Z, Jones M, et al. Integrating electronic healthcare records of armed forces personnel: developing a framework for evaluating health outcomes in England, Scotland and Wales. Int J Med Inform. 2018;113:17-25.

58. Bar-Dayan Y, Saed H, Boaz M, et al. Using electronic health records to save money. J Am Med Inform Assoc. 2013;20(e1):e17-e20.

59. de Rosis S, Barsanti S. Patient satisfaction, e-health and the evolution of the patient-general practitioner relationship: evidence from an Italian survey. Health Policy. 2016;120(11):1279-1292.

60. de Pietro C, Francetic I. E-health in Switzerland: the laborious adoption of the federal law on electronic health records (EHR) and health information exchange (HIE) networks. Health Policy. 2018;122(2):69-74.

61. Tran B, Le X, Nguyen P, et al. Feasibility of e-health interventions on smoking cessation among Vietnamese active Internet users. Int J Environ Res Public Health. 2018;15(1):165.

62. Alanazi A. Incorporating pharmacogenomics into health information technology, electronic health record and decision support system: an overview. J Med Syst. 2017;41(2):19.

63. Whitacre BE. The influence of the degree of rurality on EMR adoption, by physician specialty. Health Serv Res. 2017;52(2):616-633.

64. Tavazzi L, Ventura C. "Observational medicine": registries and electronic health recording for science and health systems governance. Eur J Heart Fail. 2016;18(9):1093-1095.

65. Barrett AK. Electronic health record (EHR) organizational change: explaining resistance through profession, organizational experience, and EHR communication quality. Health Commun. 2018;33(4):496-506.

66. Trudel M-C, Marsan J, Paré G, et al. Ceiling effect in EMR system assimilation: a multiple case study in primary care family practices. BMC Med Inform Decis Mak. 2017;17(1):46.

67. Pantaleoni JL, Stevens LA, Mailes ES, Goad BA, Longhurst CA. Successful physician training program for large scale EMR implementation. Appl Clin Inform. 2015;6(1):80-95.

68. Mohan V, Scholl G, Gold JA. Use of EHR-based simulation to diagnose aetiology of information gathering issues in struggling learners: a proof of concept study. BMJ Simul Technol Enhanc Learn. 2018;4(2):92-94.

69. Zhao C, Jiang J, Guan Y, Guo X, He B. EMR-based medical knowledge representation and inference via Markov random fields and distributed representation learning. Artif Intell Med. 2018;87:49-59.

70. Zhao JY, Kessler EG, Guo WA. Temporary removal: interprofessional communication goes up when the electronic health record goes down. $J$ Surg Educ. Epub Sept 23, 2018.

71. Rangachari P. Implementing a social knowledge networking (SKN) system to enable meaningful use of an EHR medication reconciliation system. Risk Manag Healthc Policy. 2018;11:45-53.

72. Whitacre BE. Rural EMR adoption rates overtake those in urban areas. J Am Med Informat Assoc. 2015;22(2):399-408.

73. Street RL, Liu L, Farber NJ, et al. Keystrokes, mouse clicks, and Gazing at the computer: how physician interaction with the EHR affects patient participation. J Gen Intern Med. 2018;33(4):423-428. 


\section{Publish your work in this journal}

Risk Management and Healthcare Policy is an international, peer-reviewed open access journal focusing on all aspects of public health, policy, and preventative measures to promote good health and improve morbidity and mortality in the population. The journal welcomes submitted papers covering original research, basic science, clinical and epidemiological

Submit your manuscript here: https://www.dovepress.com/risk-management-and-healthcare-policy-journal

studies, reviews and evaluations, guidelines, expert opinion and commentary, case reports and extended reports. The manuscript management system is completely online and includes a very quick and fair peerreview system, which is all easy to use. Visit http://www.dovepress.com/ testimonials.php to read real quotes from published authors. 